Predicting Tumor Responses to Gefitinib and Erlotinib

DOI: 10.1371/journal.pmed.0020021

Tyrosine kinases regulate signaling pathways that control cell growth, proliferation, motility, and other critical cellular processes. Mutations in tyrosine kinase genes can lead to abnormal kinase activity, and some tumors become dependent upon this activity for growth and survival. Thus, kinases are attractive targets for anti-cancer drugs. Examples of new kinase inhibitors include gefitinib and erlotinib, which have recently shown promise in treating non-small-cell lung cancer. Unfortunately, gefitinib and erlotinib work only in a subset of patients, and they can have severe side effects, albeit infrequently. So researchers have been trying to find ways to predict who will benefit from therapy with these drugs and who won't.

Following the work of Lynch et al. (N Engl J Med 350:2129-2139) and Paez et al. (Science 304: 1497-1500), William Pao and colleagues have previously shown that the epidermal growth factor receptor (EGFR), a tyrosine kinase, is often mutated in non-small-cell lung cancers, and that tumors that harbor such mutations are sensitive to gefitinib and erlotinib.

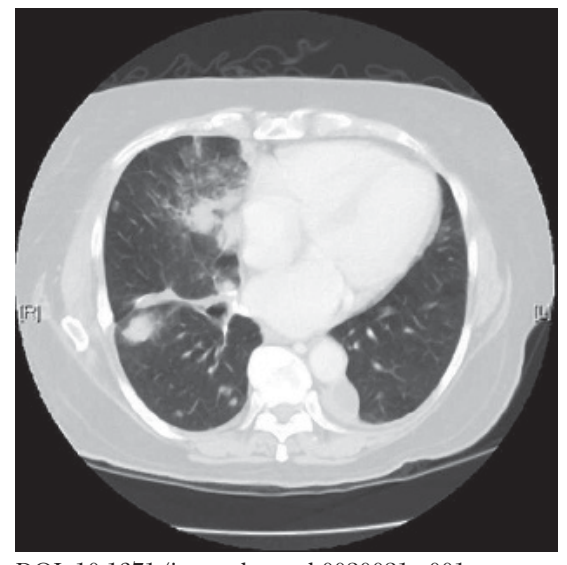

DOI: 10.1371/journal.pmed.0020021.g001

\section{Assessing lung tumors for gene mutations} could help guide therapy

In this new study, they focused on a signaling protein called KRAS, which functions downstream of many tyrosine kinases, including EGFR. The KRAS gene is also often mutated in lung cancers, but very few cancers have mutations in both EGFR and the KRAS gene. To find out whether KRAS mutations could help to predict which patients would respond to gefitinib or erlotinib, the researchers looked for mutations in EGFR and KRAS genes in 60 tumors for which sensitivity to either drug was known.

They extended their earlier findings that EGFR mutations (which were found in 17 of the tumors) were associated with sensitivity to the kinase inhibitors, and found that tumors that had mutations in KRAS (a total of nine) were refractory (i.e., did not respond) to either drug.

These results need to be validated in larger and prospective trials that use standardized mutation detection techniques. If they are confirmed, knowing the mutation status of EGFR and KRAS in tumors could help physicians decide which patients should receive gefitinib and/or erlotinib. As Inoue and Nukiwa state in a Perspective that accompanies the article, "By combining all the factors that relate to response or resistance, patients who will benefit from treatment can hopefully be identified. Undoubtedly we have taken a great step forward in molecular therapy for lung cancer treatment."

Pao W, Wang TY, Riely GJ, Miller VA, Pan $\mathrm{Q}$, et al. (2005) KRAS mutations and primary resistance of lung adenocarcinomas to gefitinib or erlotinib. DOI: 10.1371/journal. pmed.0020017

\title{
Rethinking Immunity against Pneumococcal Disease
}

DOI: 10.1371/journal.pmed.0020020

Streptococcus pneumoniae is a common bacterium that is present in the nasopharynx of many children and some adults, where it causes no harm to its carrier but can be transmitted to others. If it moves beyond the nasopharynx, however, it can cause ear infections or invasive disease, such as pneumonia or meningitis. Invasive disease from this organism occurs especially in children, the elderly, and individuals with weakened immune systems.

The protective effect of antibodies against bacterial pneumonia has been appreciated since the 1930s, when it was shown that serum therapy-the transfer of serum from an immunized animal to a patient with acute disease caused by the same bacterial strain-could reduce mortality from pneumococcal pneumonia by half. Subsequent development of vaccines based on the bacterium's polysaccharide capsule, which could protect against infection, confirmed that an endogenous antibody response can provide protection against invasive disease.

One challenge for vaccine development has been the existence of many different serotypes (the same species of bacteria but with different composition of the polysaccharide capsule). As protection usually doesn't extend to different

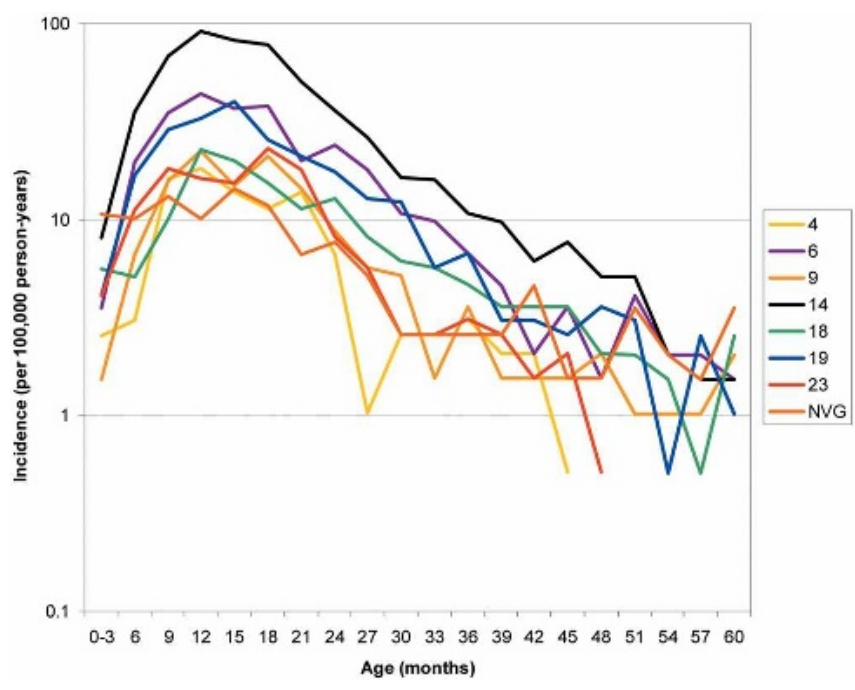

DOI: 10.1371/journal.pmed.0020020.g001

Parallel age-incidence curves for pneumococcal serotypes suggest a common mechanism of protection 
serotypes, vaccination with capsule components from different serotypes is necessary to ensure broad protection. Such vaccines have been shown to be efficient and safe. They are now recommended in many countries for infants and toddlers, and for people over 65-the two age groups in which invasive disease is most common - and for others who are at increased risk of pneumococcal disease (e.g., patients with heart, kidney, liver, or lung disease, or who have had a splenectomy).

Even without vaccination, however, most exposed individuals will never get invasive disease. Instead, they develop natural immunity against the different serotypes, though this immunity gradually declines with old age. Marc Lipsitch and colleagues wanted to understand the immunological basis of this natural immunity, and specifically whether it was due to anticapsular antibodies.

If protection from invasive disease is due to acquiring anticapsular antibodies against each of the pneumococcal serotypes, they argued, this would lead to two predictions about the age distribution of disease caused by the different serotypes in the non-vaccinated population. First, for serotypes that are more common and therefore encountered earlier in life, children should develop immunity more quickly, causing disease from these types to drop off earlier in life than disease from the less common types. Second, protection against invasive disease from a particular serotype should coincide with the acquisition of antibodies against that serotype, on both the individual and population level.

Neither prediction was borne out by the actual data the researchers analyzed, suggesting that there is more to natural immunity against pneumococcal disease than just anticapsular antibodies. The study doesn't demonstrate what the additional components are, but additional research might not just teach us about our immune system but also provide clues for further vaccine development. As the authors say, "A better understanding of the mechanisms that underlie natural immunity to pneumococcus could pave the way for the development of more effective, species-specific pneumococcal vaccines."

Lipsitch M, Whitney CG, Zell E, Kaijalainen T, Dagan R, et al. (2005) Are anticapsular antibodies the primary mechanism of protection against invasive pneumococcal disease? DOI: 10.1371/journal. pmed.0020015

\section{Meningitis and Climate in West Africa}

DOI: 10.1371/journal.pmed.0020024

Many different things combine to cause epidemics of disease. Among these factors are the characteristics of the infecting organism, the resistance of the host, and, as is increasingly realized, climatic conditions.

El Niño, the best known

climatic disturbance, is caused by a warming of the Pacific Ocean, which then affects the climate globally. Previous work has suggested that this recurring phenomenon can have a profound effect on the incidence of many diseases, including dengue, malaria, and diarrheal diseases.

In a paper in this month's PLoS Medicine, Sultan and colleagues from a climate research institute and an infectious diseases center in France looked at the relation between climate and meningitis outbreaks in Mali in West Africa, a region that every year between February and May sees devastating epidemics of meningococcal meningitis affecting up to 200,000 people. The most important recurring climatic event in this region is a dry wind, known as the Harmattan, that blows throughout the winter, causing a drop in humidity and the production of vast quantities of dust.

What the authors found was that over the years 1994-2002, the week of the onset of the yearly meningitis epidemic came at around the same time as the peak of one measure of the wind-the sixth week of the year. As Pascual and

The image has been removed due to copyright restrictions.

Dobson say in their Perspective article on this study, "Sultan and colleagues' study is exceptional in that it illustrates a clear relationship between an external environmental variable and the initiation of disease outbreaks."

How do climatic changes influence disease? In some cases, such as the role of flooding in spreading a waterborne disease, the causes are perhaps obvious, but why should a dry wind affect disease incidence? Previous works have suggested that the climate can work in a number of ways, by influencing the life cycle of both disease vectors and the diseasecausing organism, and, as here perhaps, by affecting the resistance of the host. Sultan and colleagues speculate that the drying effects of the wind on the mucous membranes could increase the chances of the organism getting established in the human host. Whatever the causes, one very useful feature of climate is that, once the patterns are understood, they can often be predicted.

A way of predicting these meningitis epidemics could be enormously useful. Sultan and colleagues looked at only a few years, but if these findings are confirmed over a longer time period, they could make preparing for an epidemic much more efficient.

Sultan B, Labadi K, Guégan JF, Janicot $S$ (2005) Climate drives the meningitis epidemics onset in West Africa. DOI: 10.1371/journal.pmed.0020006 
Epidemiological studies suggest that statins, a class of cholesterollowering drugs, may lower the risk for Alzheimer disease. The mechanism for this effect is unclear. Alzheimer disease is characterized by accumulation of amyloid deposits in the brain. These deposits are composed of amyloid-beta $(A \beta)$ peptide, a protein fragment that is cleaved off from the amyloid precursor protein APP. APP can be cleaved in two different ways. Amyloidogenic ("amyloid generating") cleavage by an enzyme called beta-secretase yields "sticky" $A \beta$ peptides that aggregate to form deposits, whereas non-amyloidogenic cleavage by alpha-secretases generates soluble peptides that do not form deposits. Studies in animal models and cell culture suggest that statins might modulate APP processing and shift the balance toward "healthy" (nonamyloidogenic) cleavage.

In their quest to understand how statins affect APP processing, Sam Gandy and colleagues focused on a molecule called ROCK1, a kinase enzyme that had recently been implicated in APP processing. The theoretical link between statins and ROCK 1 goes as follows: statins inhibit the isoprenoid pathway, isoprenoids are regulators of the enzyme
Rho, and Rho in turn activates ROCK1. And while such potential connections could be drawn for any number of molecules, Gandy and colleagues went on to test whether statins exert their effect on APP cleavage by interfering those of the statins (i.e., an increase in healthy cleavage). The same effects were seen when they transfected the cells with a dominant negative form of ROCK1 (which inactivates the normal ROCK1 molecules in the cell); this outcome shows that the pathway can influence APP cleavage. Most conclusively, when they added a version of ROCK 1 that was constitutively (always) active, they reduced basal levels and abolished statin-stimulated levels of healthy cleavage.

Taken together, these results suggest that statins influence APP processing, at least in part, by modulating the isoprenoid pathway and inactivating the ROCK1 kinase. Future studies are necessary to determine whether this mechanism is actually responsible for the apparent clinical benefits of statins. Another question worth exploring further is whether ROCK1 might be a suitable target for therapeutic interventions that aim to

with the isoprenoid/Rho/ROCK1 pathway.

Working in mouse neuroblastoma cells, they confirmed that two different statins increased healthy cleavage of APP.When they directly interfered with the isoprenoid/Rho/ROCK1 pathway by adding a drug that inhibits Rho activation, they saw effects similar to decrease harmful, and promote healthy, cleavage of APP.

Pedrini S, Carter TL, Prendergast G, Petanceska S, Ehrlich ME, et al. (2005)

Modulation of statin-activated shedding of

Alzheimer APP ectodomain by ROCK. DOI: 10.1371/journal.pmed.0020018

\section{Modeling the HIV Epidemic in Africa}

DOI: 10.1371/journal.pmed.0020023

The HIV epidemic is continuing to grow, year by year. According to the Joint United Nations Programme on HIV/AIDS (UNAIDS), in 2004 there were more people living with the virus than ever before, and in the same year more people than ever before died of it. So, although in the developed world HIV/AIDS is a controllable disease, one with which a treated person might expect to have a near normal lifespan, in much of the rest of the world AIDS is still a death sentence. Despite the fact that the cost of AIDS medicine has come down to around $\$ 150$ per year in the developing world - a much lower cost than previously-the drugs are still unavailable to the vast majority of patients. What is more, every infected person has the chance of infecting many others. Although huge sums of money have been poured into combating HIV/AIDS-around US\$4.7 billion in 2003-UNAIDS estimates this amount is less than half of what is required by 2005 , and only a quarter of what will be required by 2007 , to mount a comprehensive response to AIDS in low-income and middle-income countries. One of the real dilemmas, therefore, of HIV/AIDS policy is deciding whether it is better to concentrate resources on prevention of infections or on treatment of infected individuals. Each approach has ramifications for the other, as shown by the experience in some developed countries, where an increase in availability of treatment has been accompanied by an increase in risk behavior.

An analysis by Joshua Salomon and colleagues in this month's PLOS Medicine suggests that trying to concentrate on one or the other of these alternatives is a false dichotomy, and that not integrating the two approaches could have a catastrophic effect on the global toll of HIV/AIDS by 2020. In this theoretical paper the authors analyze the epidemic in sub-Saharan Africa (where three-fourths of deaths from AIDS occur). With no change in current levels of prevention and care, it is predicted that there will be 3.7 million new HIV infections and 2.6 million adults dying of AIDS in this region each year within the next two decades. 
The authors predicted that concentrating on prevention alone could decrease yearly infections by half, and that concentrating on treatment could decrease yearly infections by $6 \%$. However, combining both approaches could yield substantially greater benefits than the sum of the two alone-lowering projected new infections by $74 \%$ and projected annual mortality by half. These percentages translate into 29 million new infections and 10 million deaths averted between 2004 and 2020.

The challenge now is obviously how to put these policy suggestions into practice. The current World Health Organization treatment target of having three million people on antiretroviral therapy by the end of 2005 (the " 3 by 5 " objective) provides a yardstick for only one part of the equation. The authors comment that the mobilization of communities that will be needed to achieve the 3 by 5 objective should also be harnessed for prevention, and that those who teach prevention must also be allowed to get care for those infected. As the authors say, only by doing so "will we at last move from slogans to impact."

Salomon JA, Hogan DR, Stover J, Stanecki KA, Walker N, et al. (2005) Integrating HIV prevention and treatment: From slogans to impact. DOI: 10.1371/journal.pmed.0020016

\section{New HIV infections (millions)}

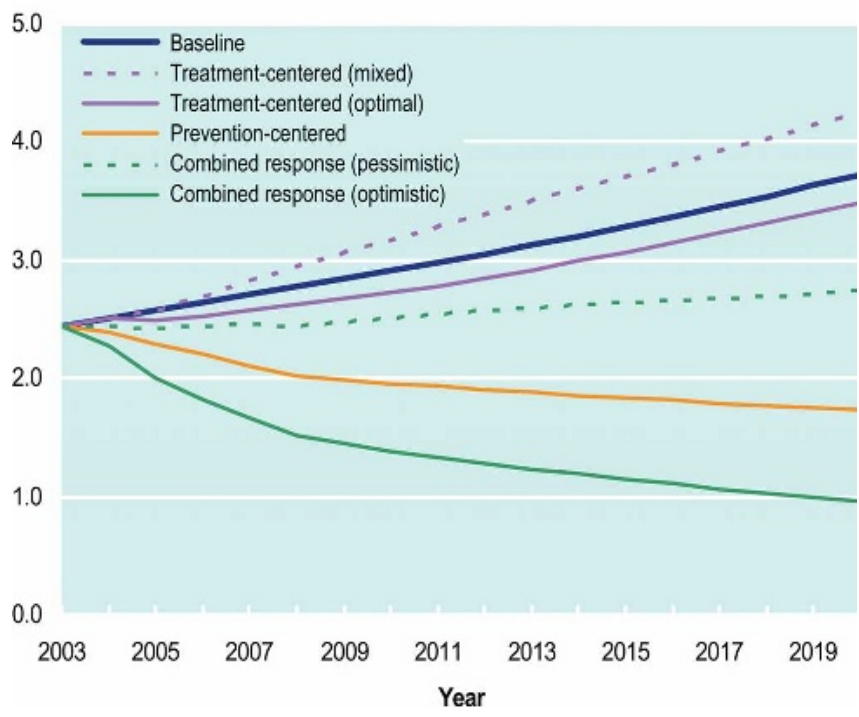

DOI: 10.1371/journal.pmed.0020023.g001

The best strategy is to combine prevention and treatment 